\title{
Application Specific Hardware Design Simulation for High Performance Embedded System
}

\author{
Ravi Khatwal \\ Research scholar \\ Department Of Computer science, \\ Mohan LaL Sukhadia University, \\ Udaipur, India.
}

\author{
Manoj Kumar Jain, Ph.D \\ Associate Professor, \\ Department Of Computer science, \\ Mohan LaL Sukhadia University, \\ Udaipur, India.
}

\begin{abstract}
Application specific simulation is challenging task in various real time high performance embedded devices. In this study specific application is implemented with the help of Xilinx. Xilinx provides SDK and XPS tools, XPS tools used for develop complete hardware platform and SDK provides software platform for application creation and verification. Xilinx XUP-5 board have been used and implemented various specific Applications with hardware platform. In this study the base instruction set with customized instructions, supported with specific hardware resources are analyzed.
\end{abstract}

\section{Keywords}

Xilinx, virtex-5 FPGA board, simulation, hardware and software design, Xilinx Platform Studio.

\section{INTRODUCTION}

In co-design methodology, the hardware and software components for an embedded system are designed jointly. Each of the hardware and software components designed using appropriate tools (hardware synthesis, code generation and hardware-software co-simulation tools). In ASIP design technology hardware can be design according for specific application. Xilinx SDK provides software environment used for various specific application verification and creation. Kucukcakar, K. [1] proposed a unique architecture and methodology to design ASIPs in the embedded controller domain by customizing an existing processor instruction set and architecture. Jain, M.K., Balakrishnan, M., Kumar, A. [2] proposed survey in ASIP area and identifies some issues which need to be addressed. Hartmann, M., Raghavan, P., Agrawal,P., Dehaene, W. [3] proposed a design method for memristor-based (ReRAM) memory architectures for embedded processors to address the effects caused by longer write latencies. Sharma, A., Sutar, S., Sharma, V.K., Mahapatra, K.K. [4] designed an ASIP using language for instruction-set architecture (LISA) and designed processor has optimized instructions for the image enhancement application in spatial domain. Fathy, A.,Isshiki, T.,Li, D., Kunieda, H. [5] presented a complete framework for searching for Application specific special instruction patterns based on tree scan algorithm while tweaking it to fit real applications. J. Qiu, X. Gao, Y. Jiang, X. Xiao [7] proposed a hybrid simulation framework which improves the previous simulation methods by aggressively utilizing the host machine resources. H. M. Hassan, K. Mohammed and A. F. Shalash [8] presented an ASIP design for a discrete Fourier transform (DFT)/discrete cosine transform (DCT)/finite impulse response filters (FIR) engine.

\section{EMBEDDED DEVELOPMENT KIT}

Xilinx [6] provides Embedded Development Kit (EDK) (see figure 1) tools to design a complete embedded processor system for implementation in a Xilinx FPGA device. Xilinx Platform Studio (XPS) is the development environment used for designing the hardware platform and Software Development Kit (SDK) is an integrated development environment used for $\mathrm{C} / \mathrm{C}++$ embedded software application creation and verification. Embedded Development Kit also provides ISE Plan ahead is used to design custom Memory and processor configuration according to specific application. Xilinx EDK provides XPS, SDK and ISE tools for simulation analysis.

\subsection{XPS}

XPS provides hardware system development environments and provides Specification of the microprocessor, peripherals, and the interconnected components, along with their respective detailed configuration.

\subsection{SDK}

SDK provides software development environments and also used for developing standalone application.

\subsection{ISE Design}

ISE design used for verifies the correct functionality of Hardware Description Language (HDL), RTL and schematic design. 


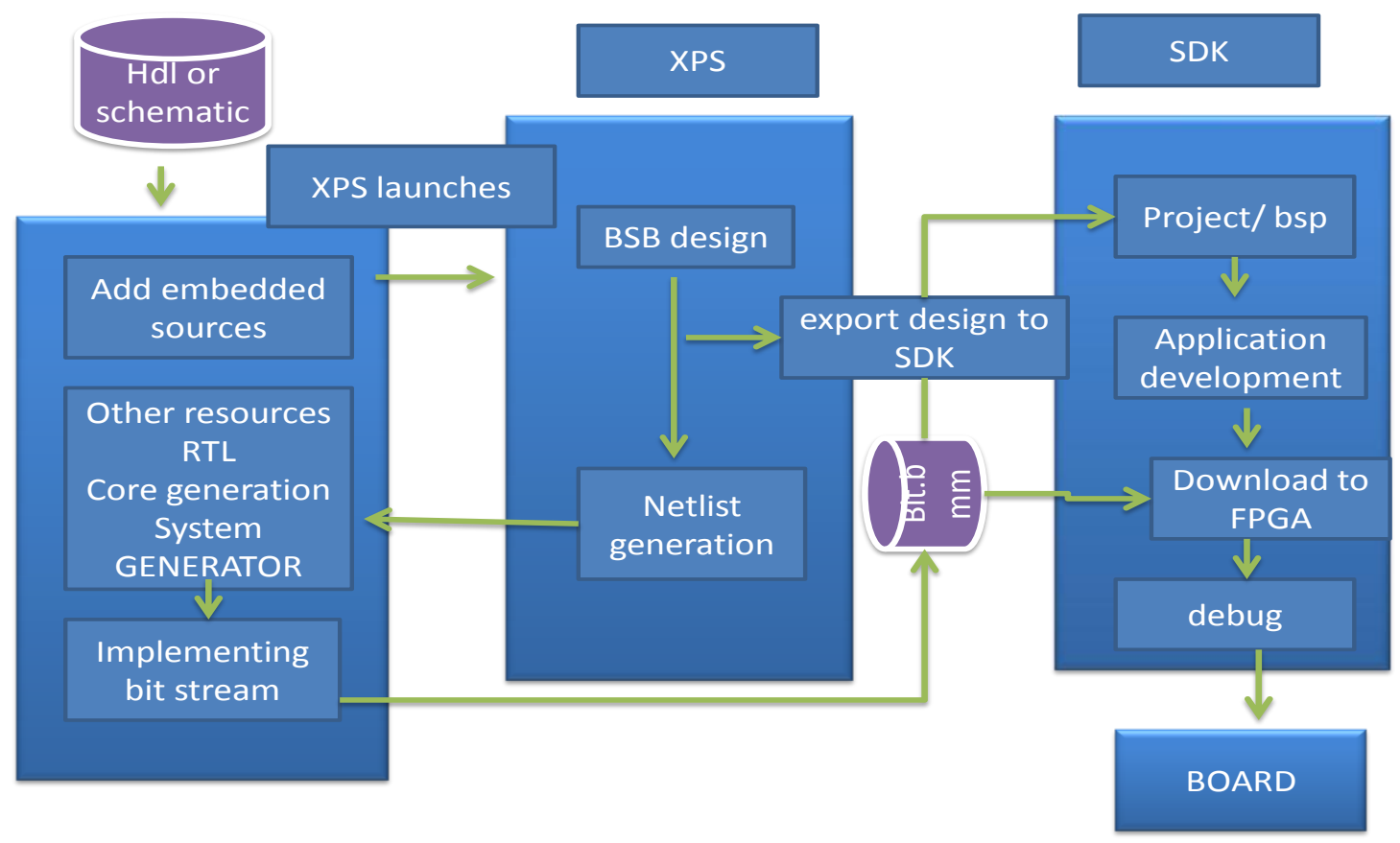

Fig 1: EDK Design flow analysis

\section{XILINX PLATFORM STUDIO (XPS)}

Xilinx Platform Studio (XPS) provides an interactive development environment that allows specifying all aspects of our hardware platform and XPS also maintains the hardware platform description in a high-level form, known as the Microprocessor Hardware Specification (MHS) file. XPS used to synthesizes the MHS source file into netlists used for the FPGA place and route process. The MHS file [6] is integral to our design process and contains all peripheral instantiations along with their parameters. The MHS file also defines the configuration of the embedded processor system and includes information on the bus architecture; peripherals, processor, connectivity, and address space etc. One of the supported embedded processor development boards available from Xilinx [6] has been selected for target architecture.

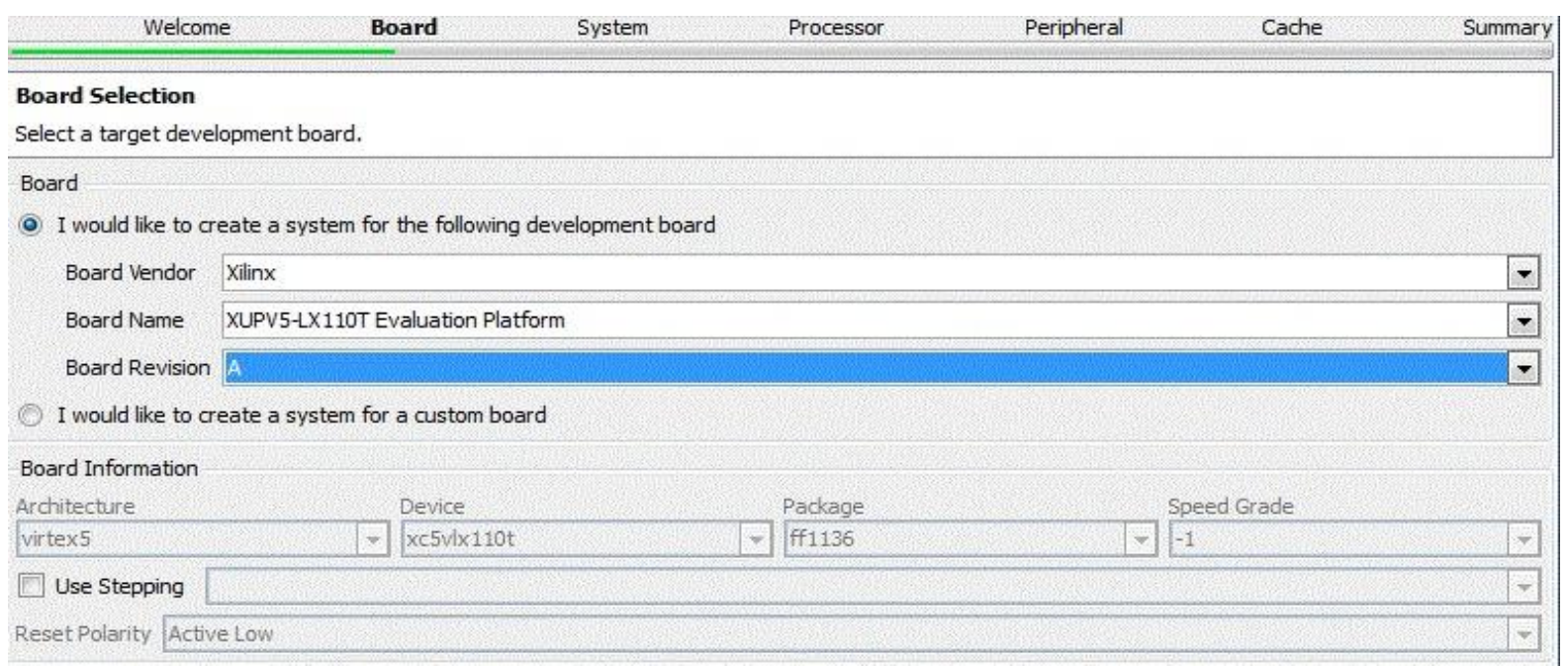

Fig 2: xup-5 fpga board selection

In XPS platform have configured selected XUP-5 FPGA platform (see figure2) for simulation and XUP-5-LX110T board utilizes Xilinx virtex5 XC5VLX110T-FF1136 device. A complete embedded processor system implemented within a Xilinx FPGA device. Hardware platform contains one or more processors, along with a variety of peripherals and memory blocks. The behavior of each processor or peripheral core can be easily customized (see figure $3,4 \& 5$ ). Hardware platform is ultimately implemented in the FPGA and 
Instruction and Data cache sized change according to desire application (see figure 6\&7).

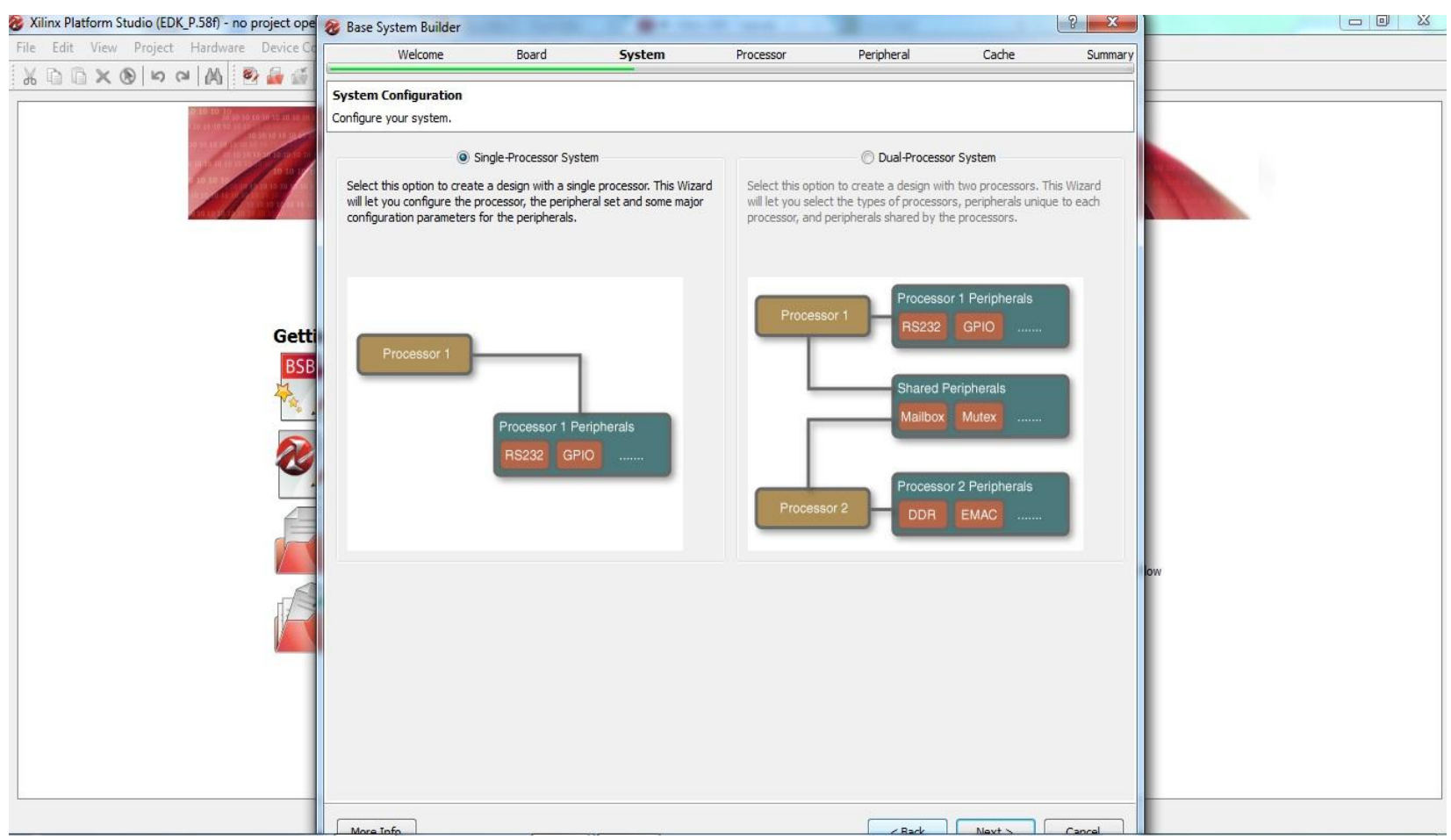

Fig 3: Processor configuration

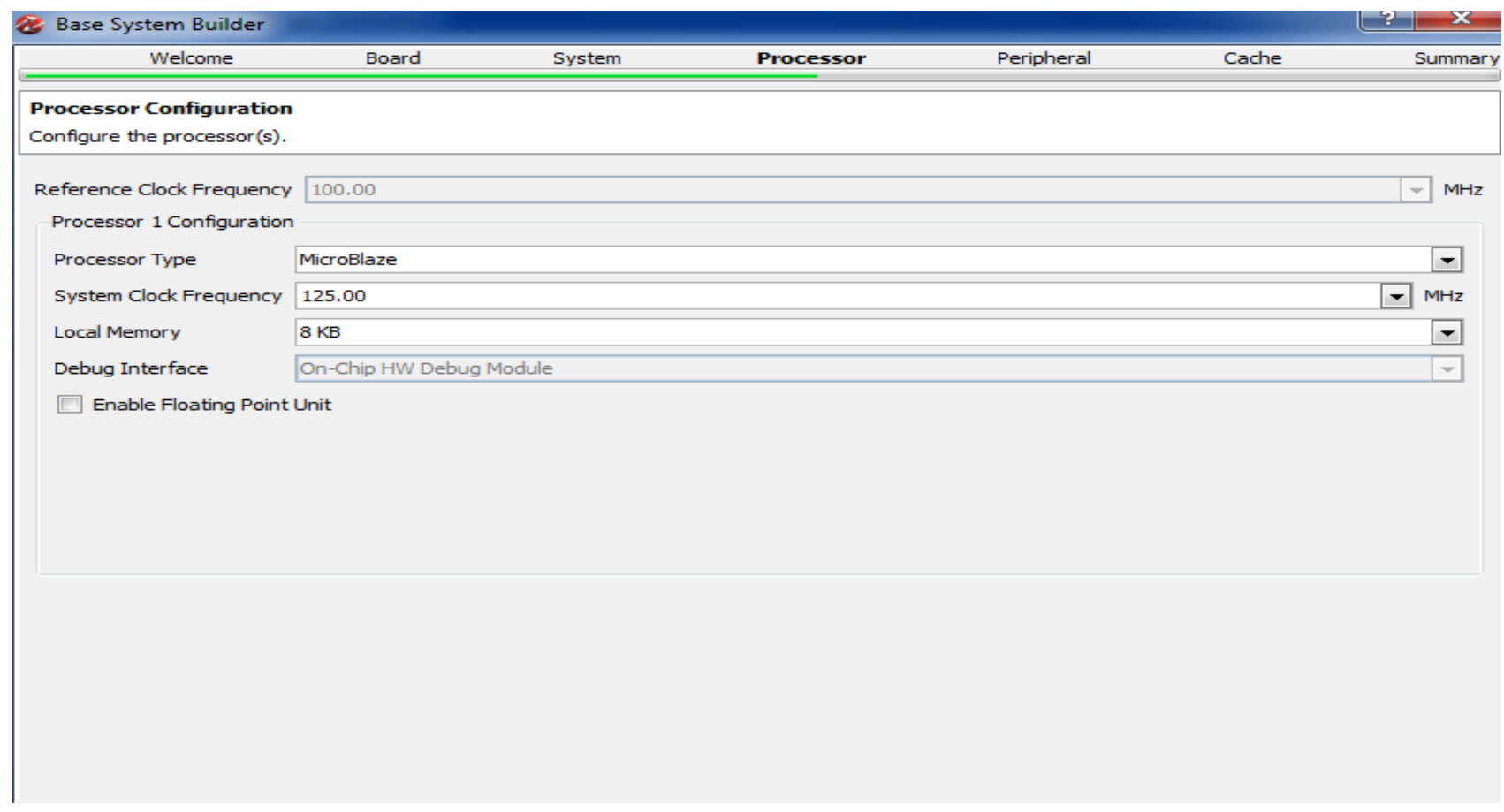

Fig 4: Micro blaze processor configuration 


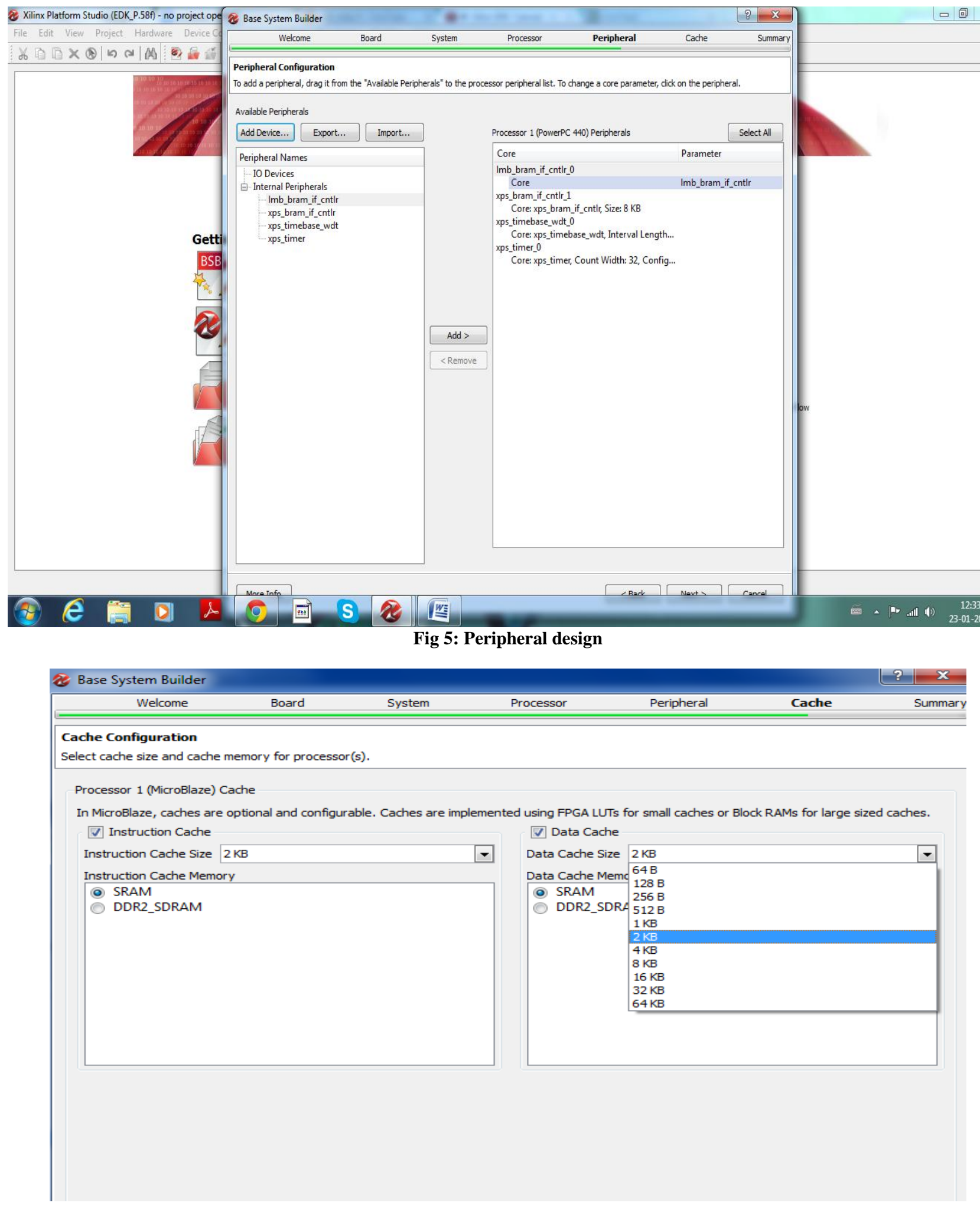

Fig 6: I- Cache configuration 


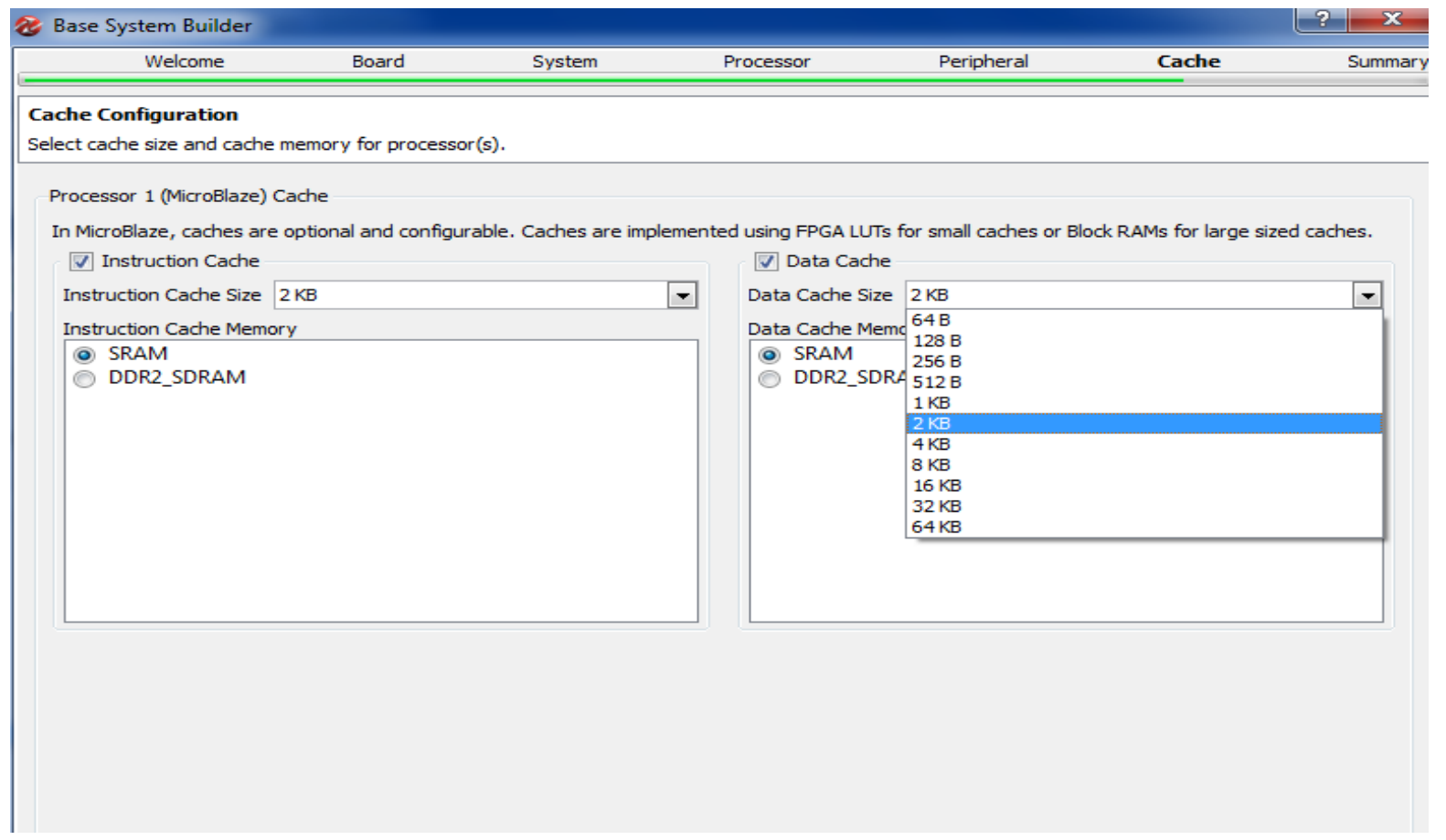

Fig 7: D-Cache configuration

XPS (see figure 8) used the Base System Builder, and it generated a bit stream for the FPGA. Hardware platform is exported to the Software Development Kit (SDK) platform. The hardware platform contains the XML-based hardware description file, the bit stream file, and the BMM file. XPS System Assembly View allows for view and configures the system block elements. XPS under implement flow; it generated net list implements, the design using the Xilinx backend, synthesis and route to create the final Netlist. The architectural design of selected processor and memory (see figure 9 \& 11) and their VHDL configuration used for specific application (see figure $10 \& 12$ ).

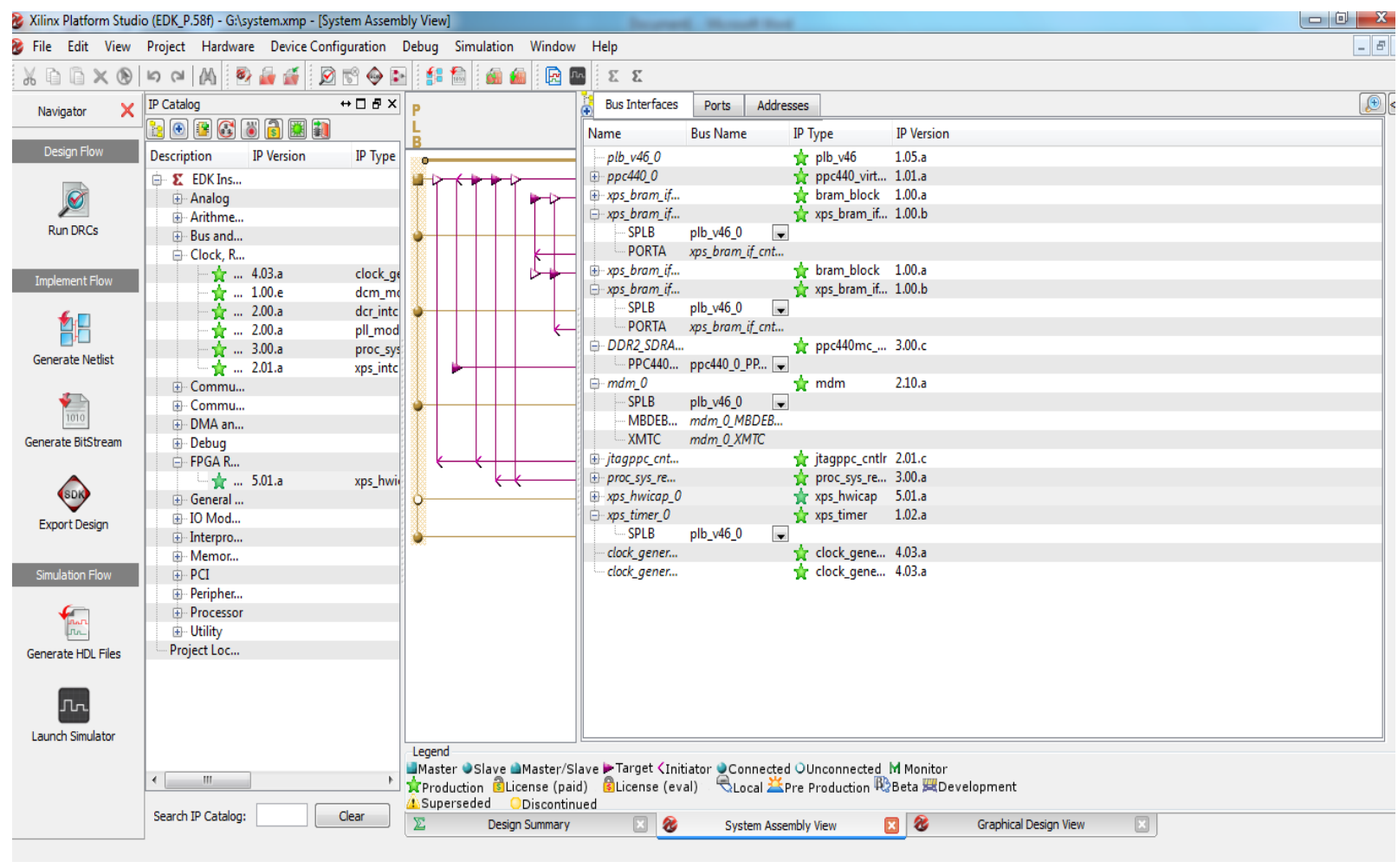

Fig 8: XPS system assembly view analysis 


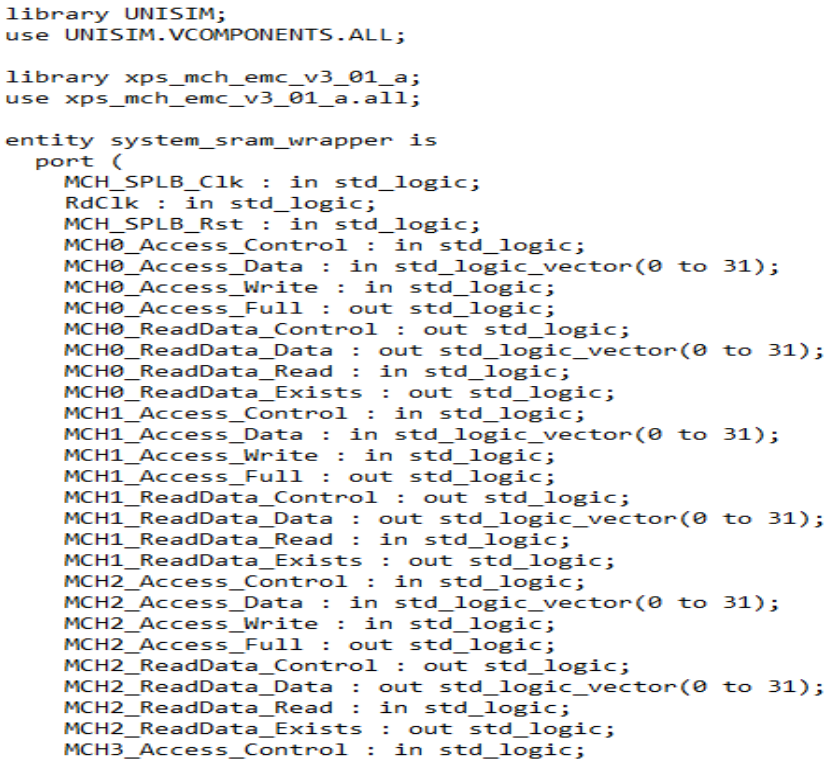

Fig 9: Memory VHDL configuration files

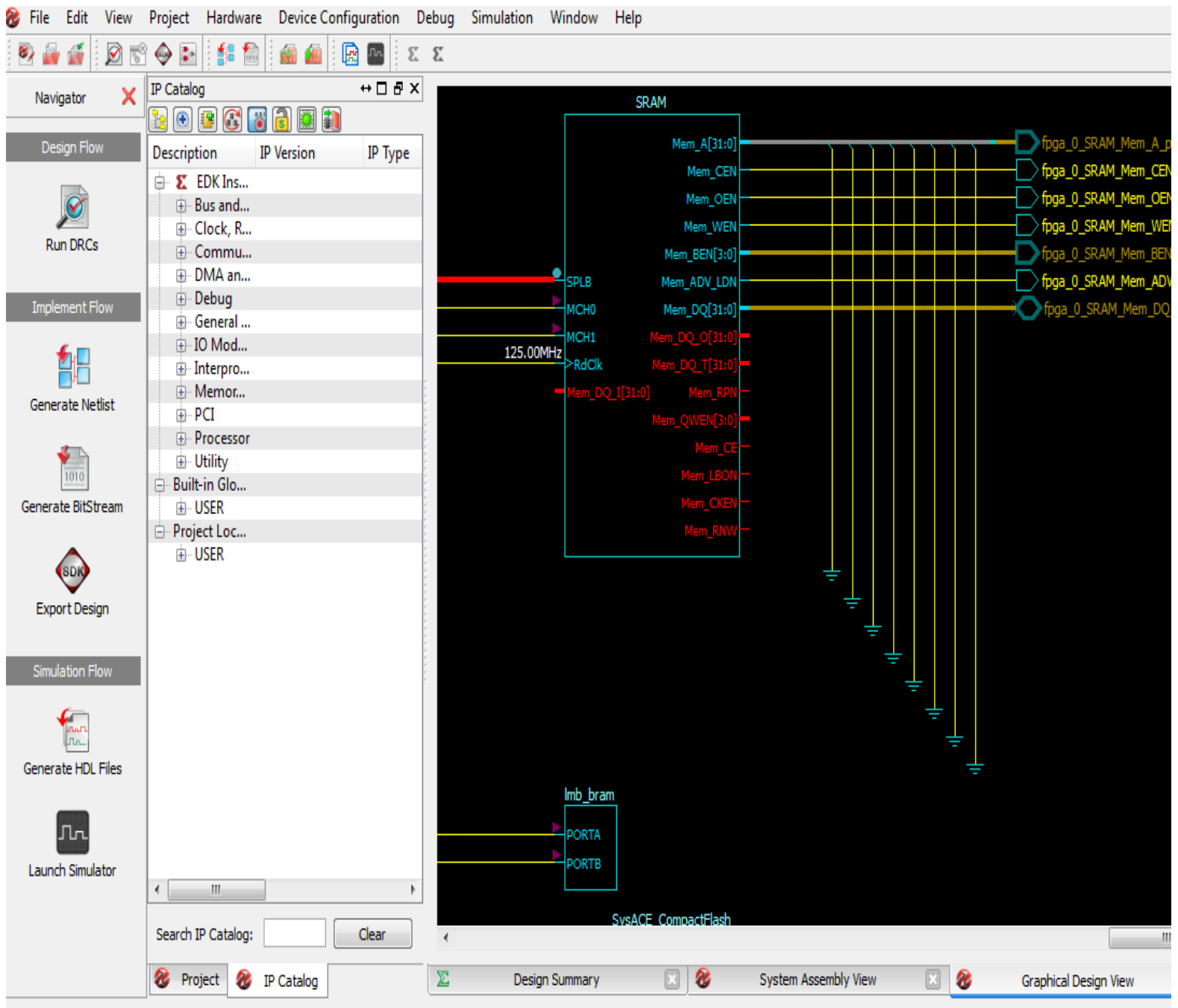

Fig 10: Graphical Design of Memory 


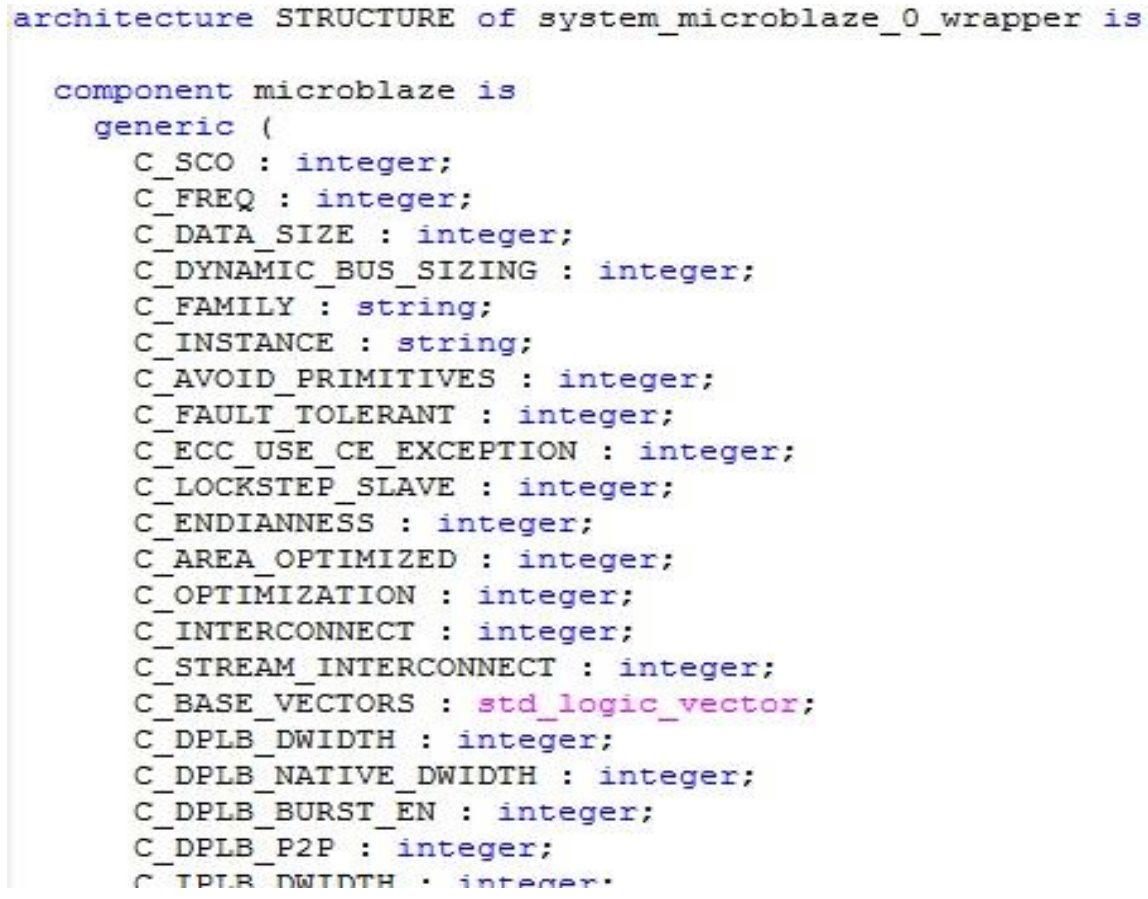

Fig 11: Processor configuration VHDL file

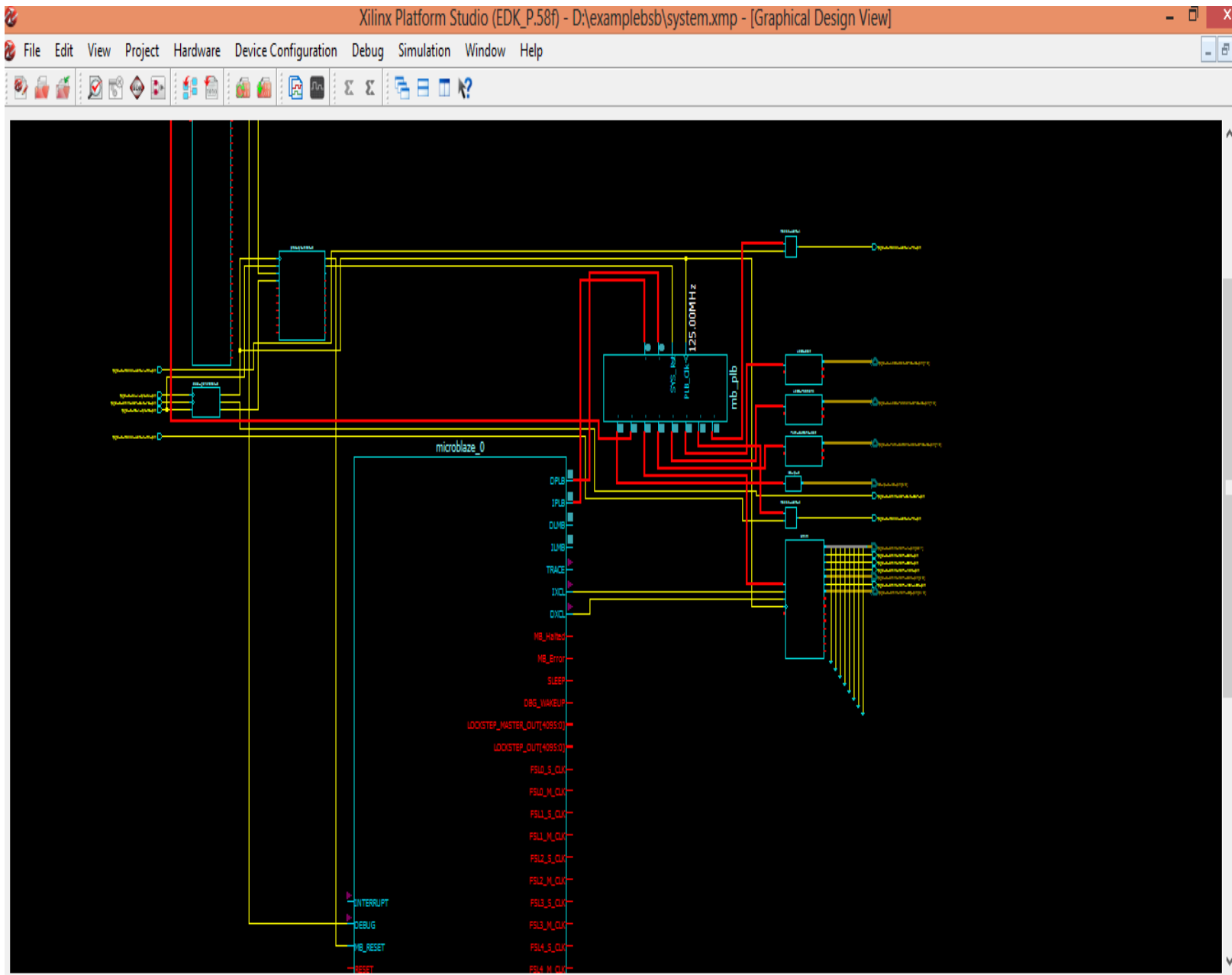

Fig 12: Microblaze processor design 


\section{SOFTWARE DEVELOPMENT KIT}

XPS design is exported to the Software Development Kit (SDK) platform. Various Software applications must link against or run on top of a given software platform, using the specific provided Application Program Interfaces (APIs).
Software Part (sdk) contains one or more source files, along with the necessary header files, used for compilation and generation of a binary output (.elf) file (see figure15).

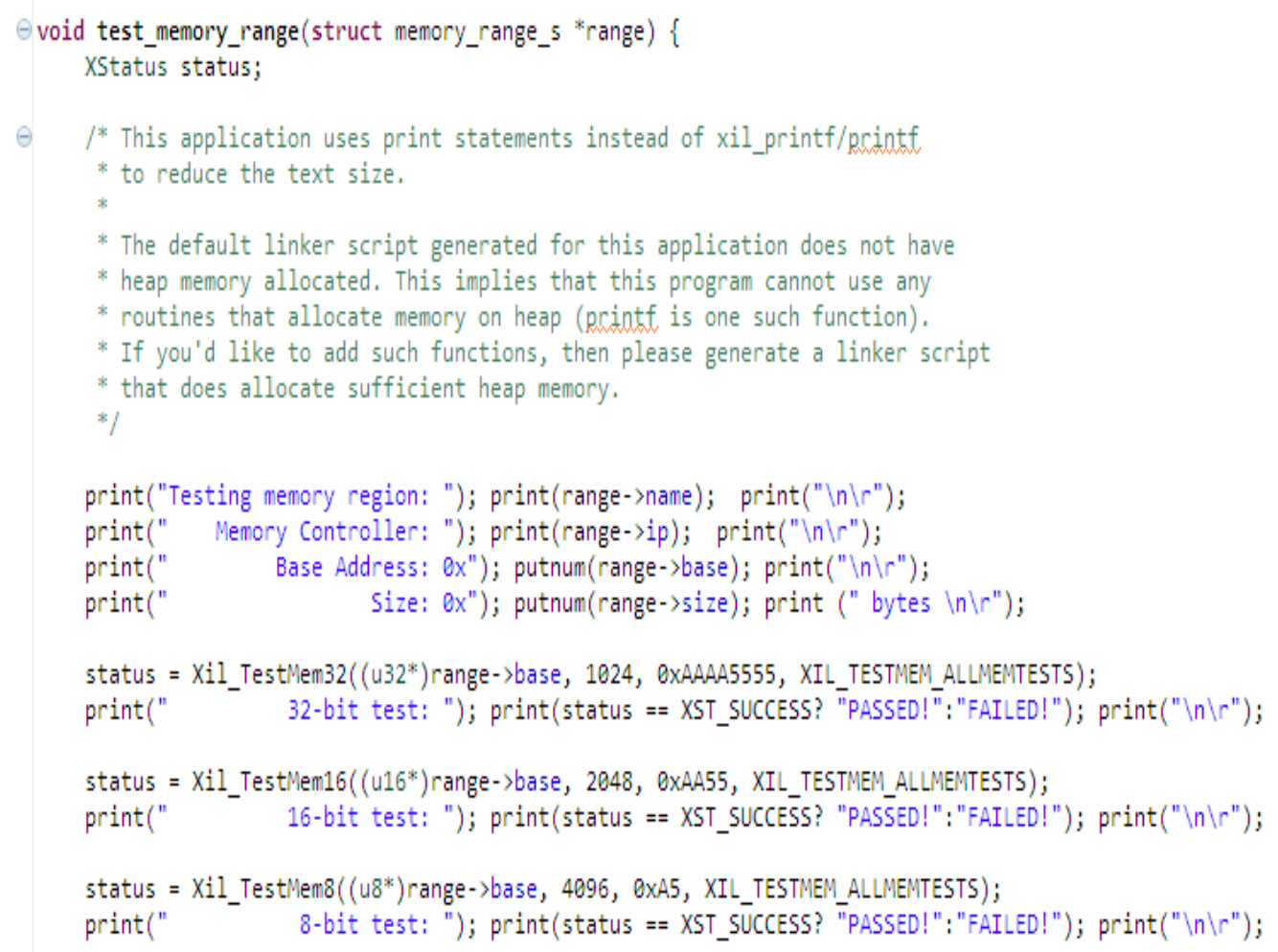

Fig 13: Memory testing application analysis on SDK platform

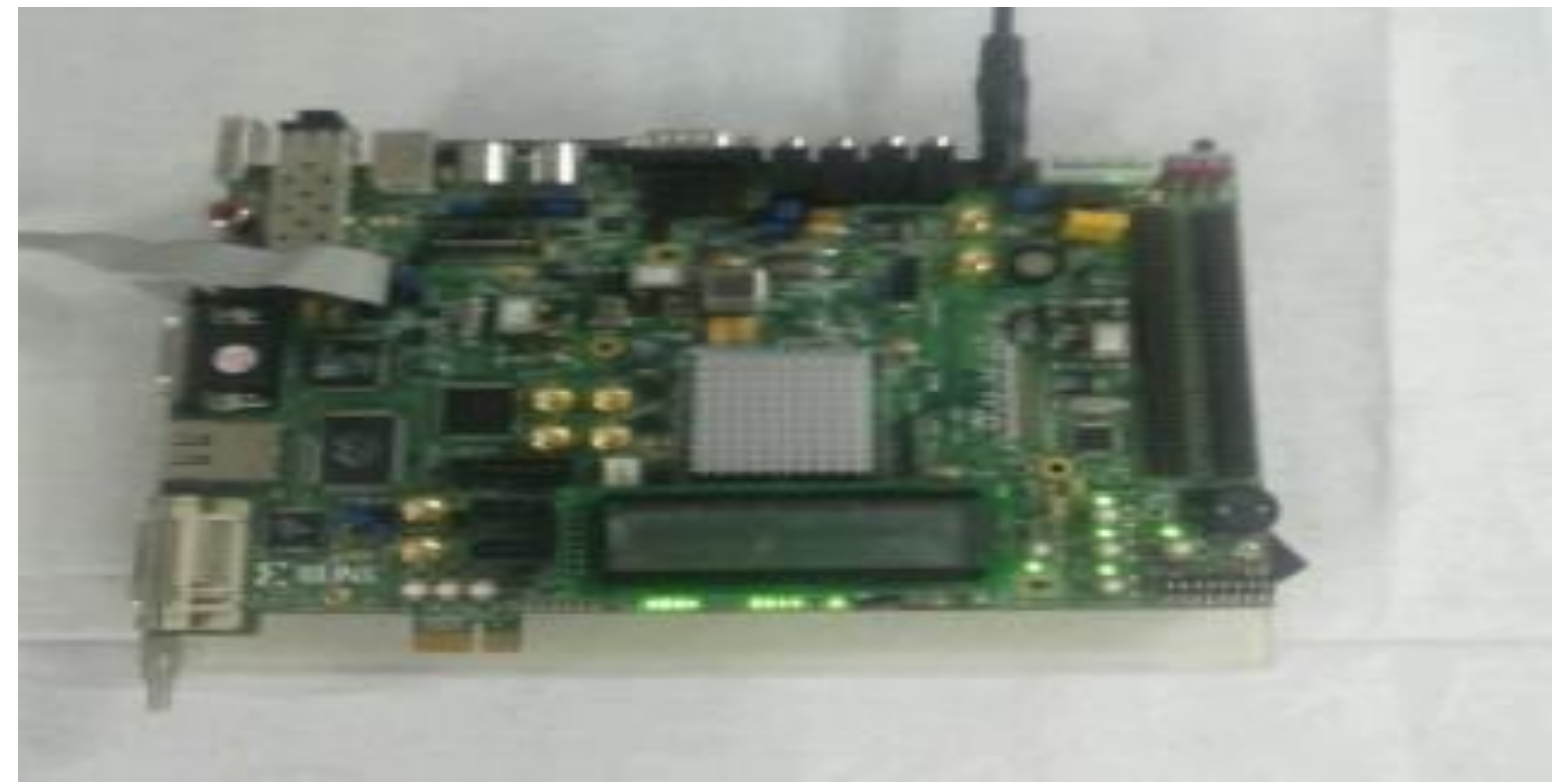

Fig 14: Xup-5 FPGA Board 


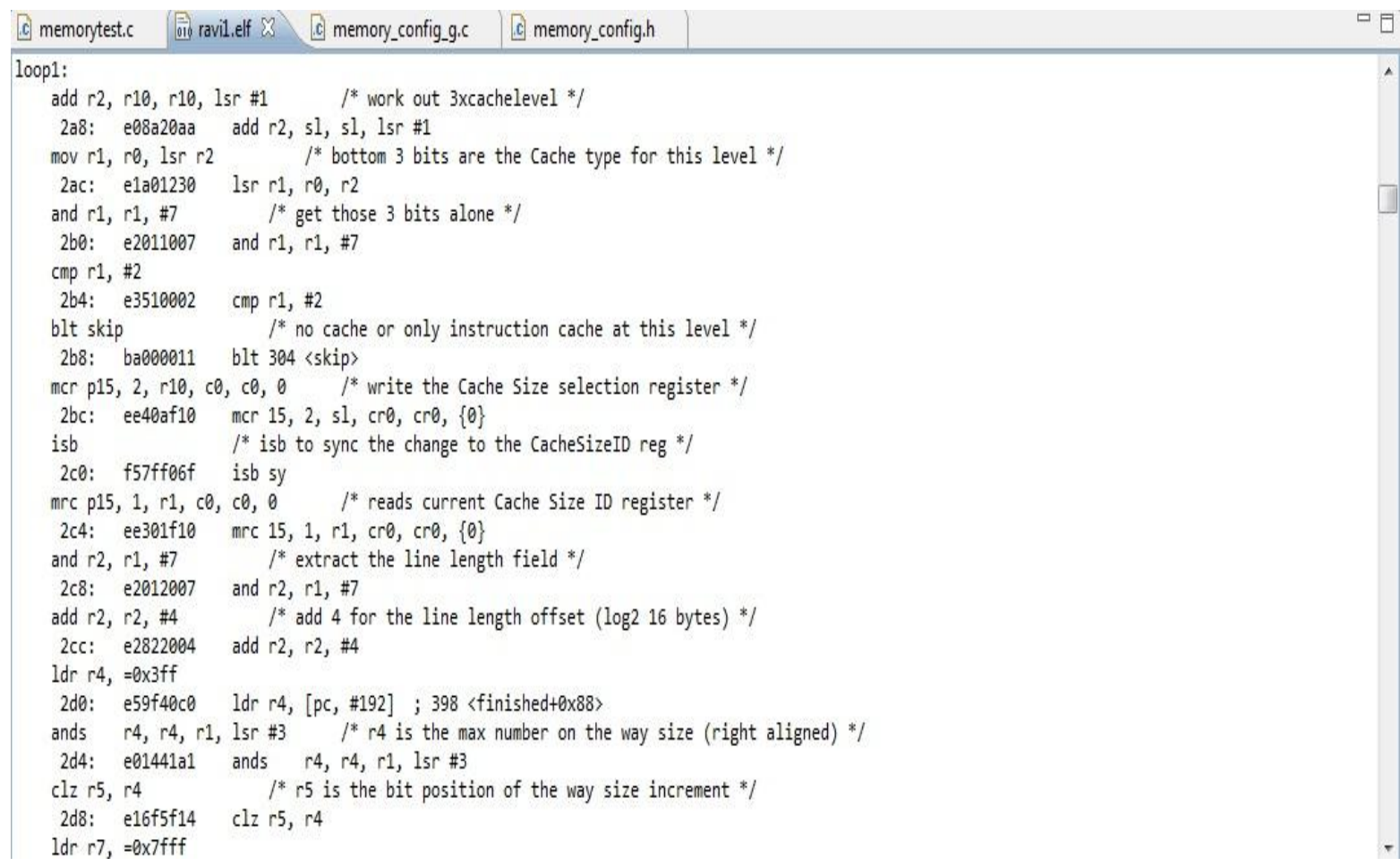

\section{Fig 15: ISA Simulation result of specific application}

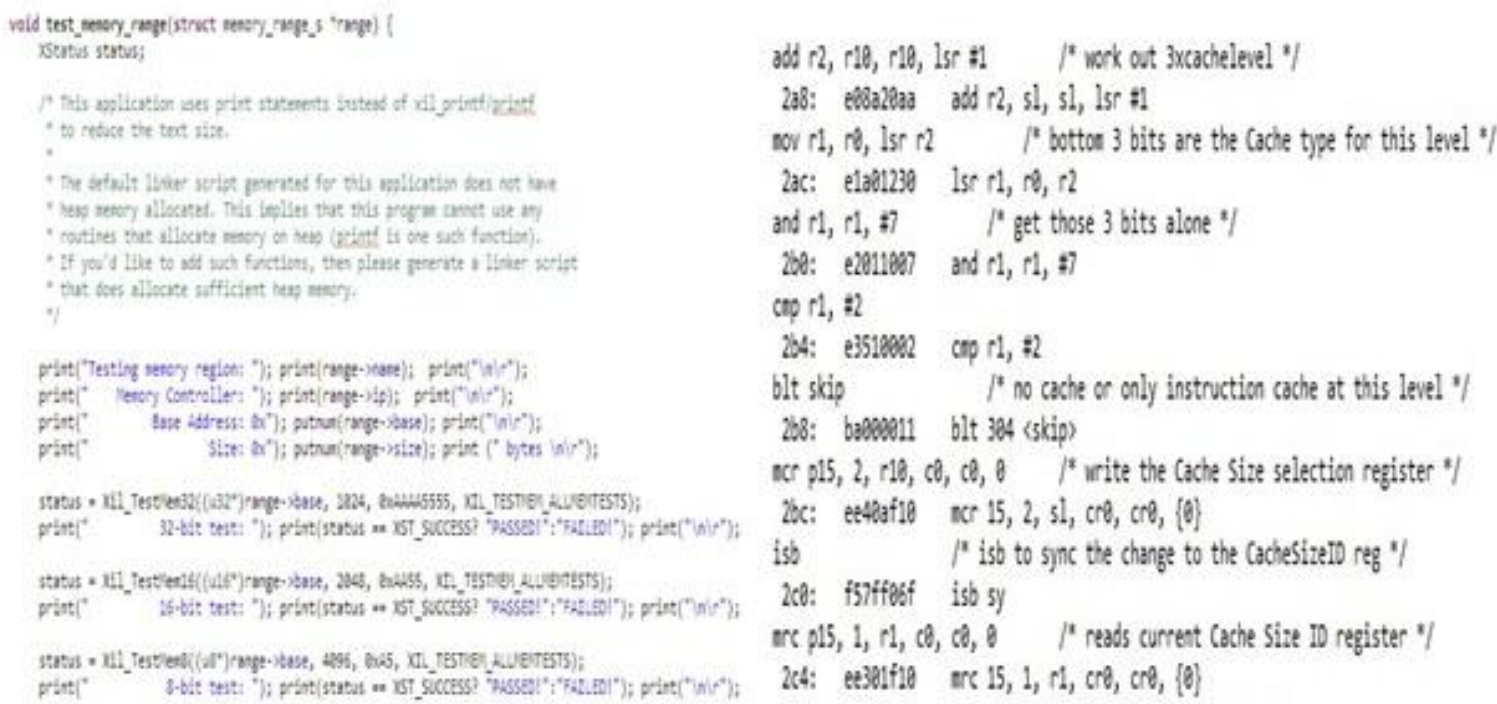

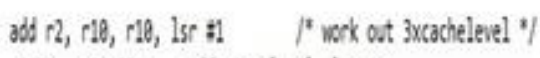

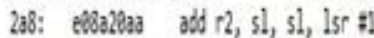

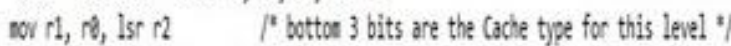

2ac: ela0:1230 lso $\mathrm{r}, \mathrm{r} 8 \mathrm{r}, \mathrm{r}$

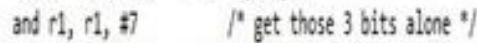

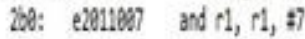

$\operatorname{cop} \mathrm{rl}_{1}, 2$

264: e3518002 cop $\mathrm{rl}$, ;2

blt skip I* no cache or only instruction cache at this level '/

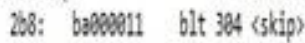

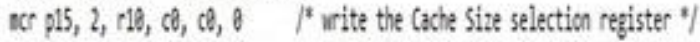

Zcc: eetastie acr 15, 2, sl, $\mathrm{cre}, \mathrm{cre},[0\}$

isb "isb to sjnc the change to the cachesizell reg "

2ce: ififfesf iso $5 y$

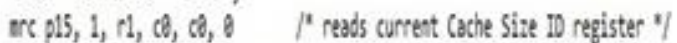

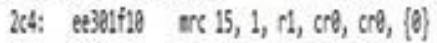

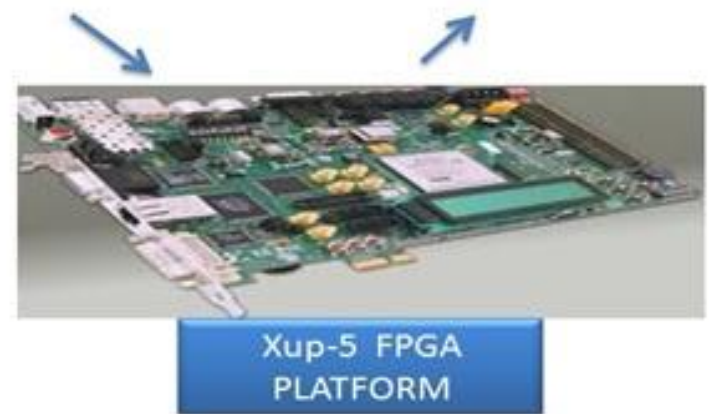

Fig 16: Application specific simulation analysis 


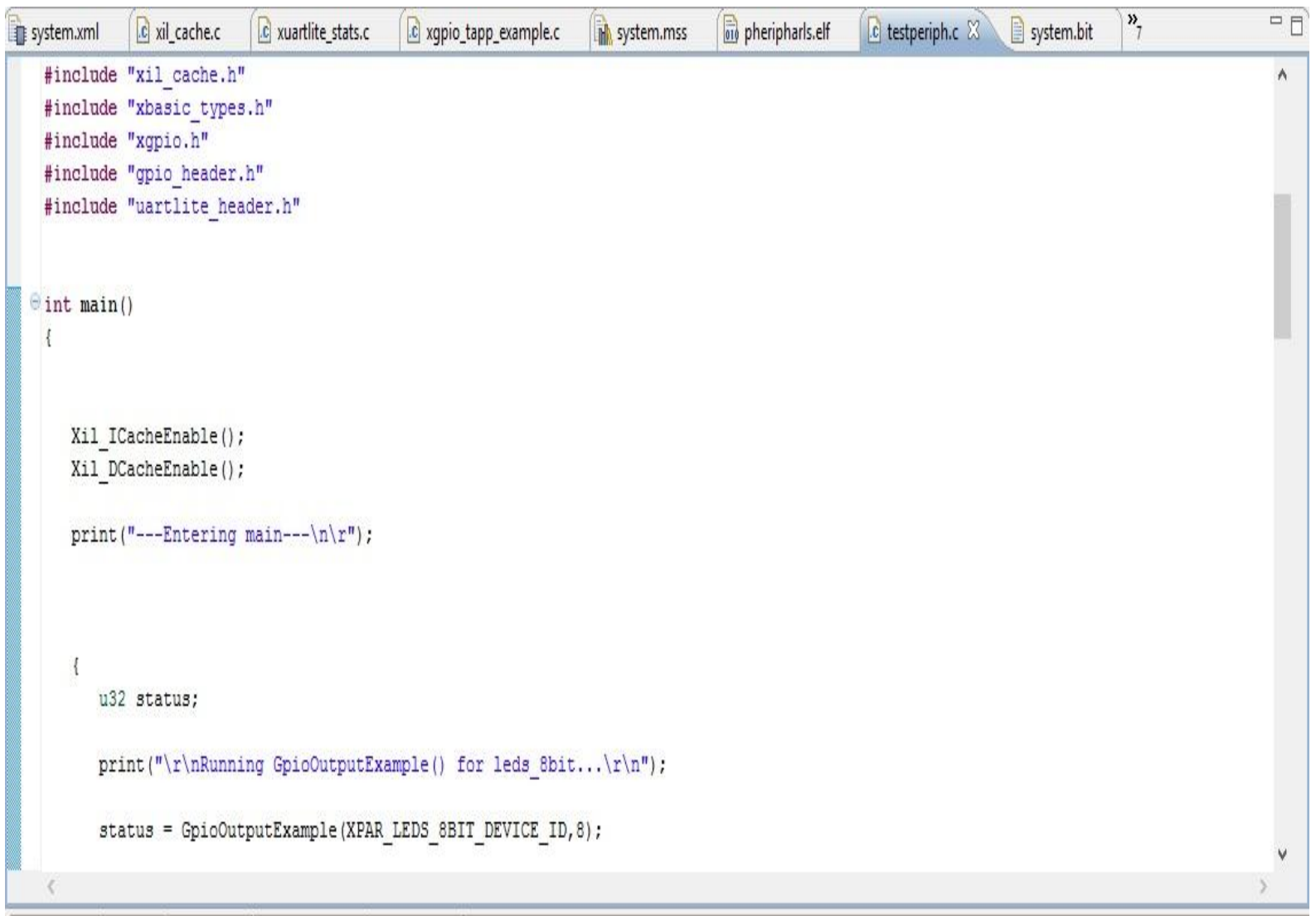

Fig 17: Peripheral test application



Fig 18: Peripheral appplication Simulation results 


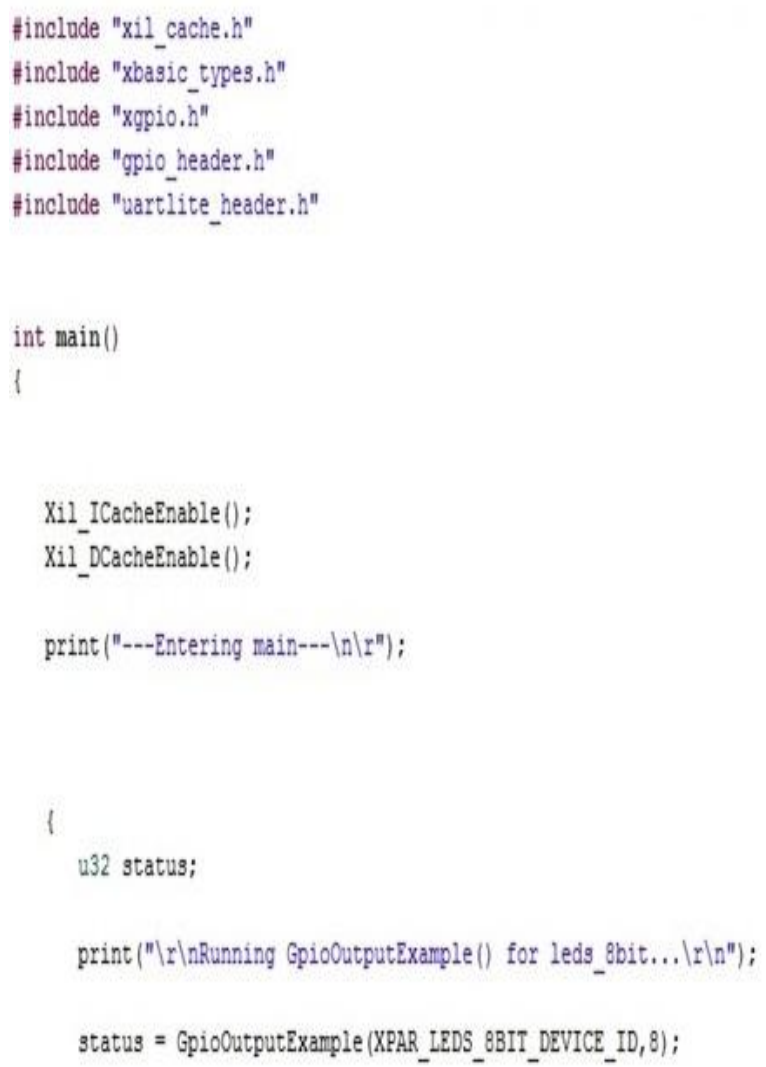

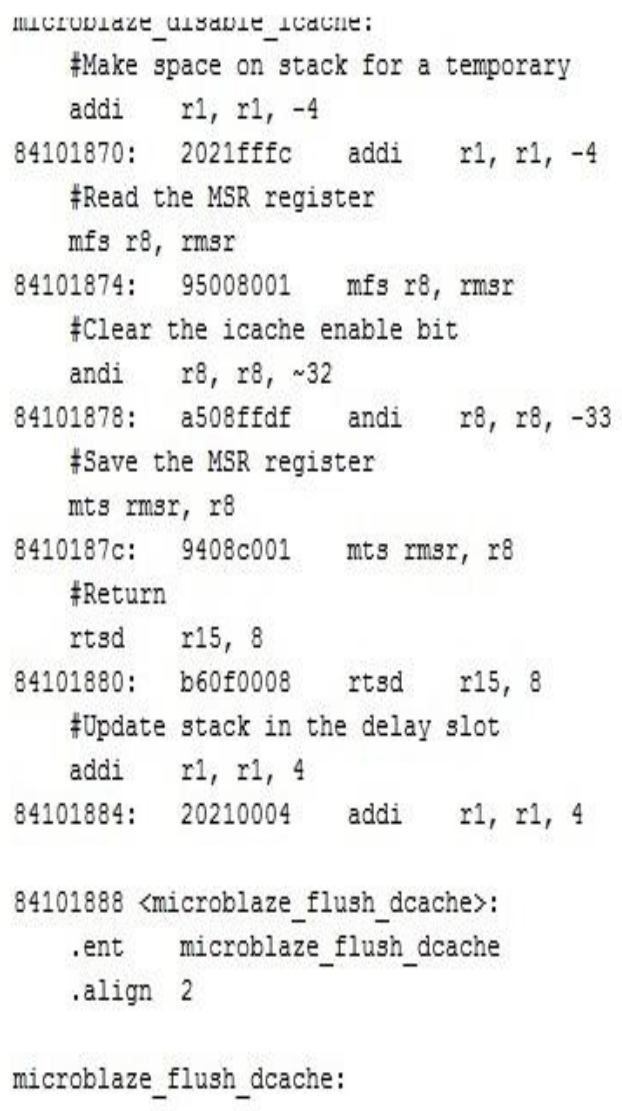

\section{APPLICATION}
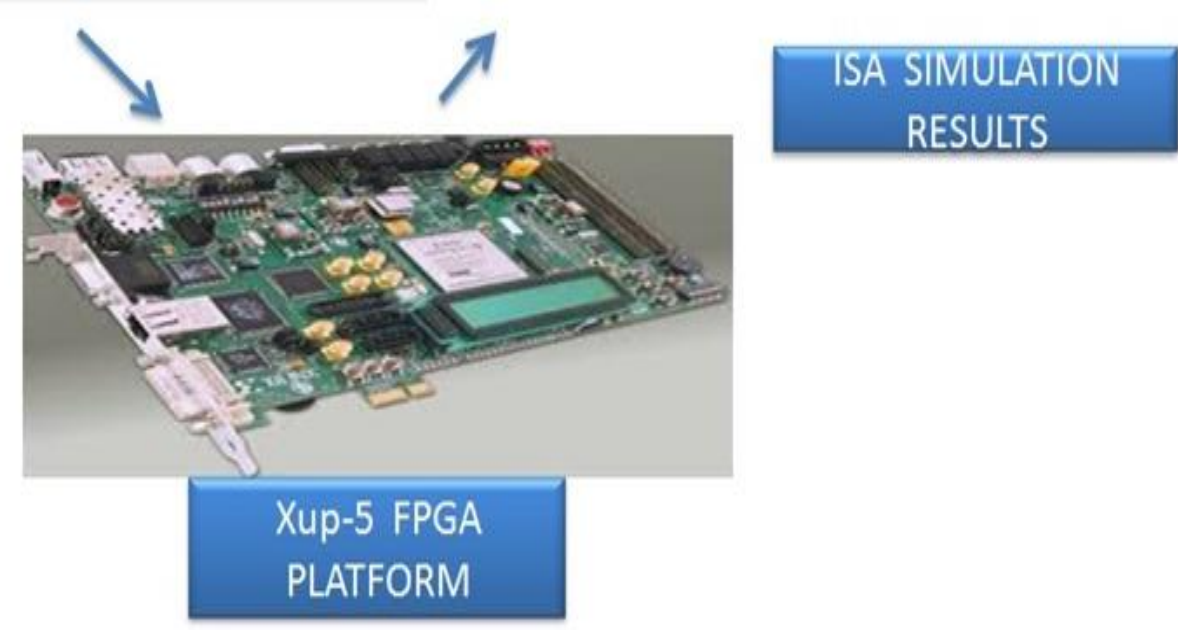

Fig 19: Peripheral application simulation analysis with xup-5 FPGA BOARD

Memory testing application (see figure 13) used on specific target hardware (see figure 14). SDK examined the hardware specification file and tested the memory application with xup5 FPGA board. It generates the elf file and elf contains the ISA behavior for specific application. After the simulation process (see figure 16) we get efficient ISA result (see figure 15). Peripherals testing another application (see figure 17) is used for simulation. It generates the simulation results (see figure 18). The simulation process is to analyze on xup-5 fpga device with custom hardware environment (see figure19).

\section{CONCLUSION}

Our main focus in this paper is application specific simulation analysis with specific hardware environment. Hardware resources like processor; memories etc. easily implemented and analyzed custom instruction set behavior for specific application. Various standard applications analyzed on xup-5 FPGA board with specific hardware environments. The ISA behaviour is also analyzed. After this simulation process application specific results are used for high performance embedded system.

\section{REFERENCES}

[1] Kucukcakar, K. An ASIP design methodology for embedded systems. In proc of: Hardware/Software Codesign, 1999.

[2] Jain, M.K., Balakrishnan, M., Kumar, A., "ASIP Design Methodologies: Survey and Issues", VLSI, 14th 
International Conference on VLSI Design (VLSID '01), 2001.

[3] Hartmann, M., Raghavan, P., Perre, V. D., L., Agrawal, P., Memristor-Based (ReRAM) Data Memory Architecture in ASIP Design, IEEE, Digital System Design (DSD), 2013,Euromicro Conference. pp.795 798.

[4] Sharma, A., Sutar, S., Sharma, V.K., Mahapatra K.K. An ASIP for image enhancement applications in spatial domain using LISA,2011, pp.175-179.

[5] Fathy A., Isshiki T.,Li D., Kunieda H. Custom Instruction Synthesis Framework for Application
Specific Instruction-Set Processor with HW, IC-ICTES in Ayutthaya, 2014.

[6] Xilinx tool Available from http//www.xilinx.com.

[7] Qiu, J., Gao, X., Jiang, Y., Xiao, X. An ultra-fast hybrid simulation framework for ASIP, Electronics, Circuits and Systems (ICECS), 2011 18th IEEE International Conference. pp.711- 714 .

[8] Hassan, H. M., Mohammed, K. and Shalash, A. F. Implementation of a reconfigurable ASIP for high throughput low power DFT/DCT/FIR engine, engine EURASIP Journal on Embedded Systems, 2012. 